\title{
Effect of Copolymer Composition on Thermodynamic Interactions in Blends Containing a Diene-Olefin Copolymer and a Polyolefin
}

\author{
Jialin Qiu, ${ }^{a}$ Xuejian Chen, ${ }^{a}$ Carlos R. López-Barrón, ${ }^{b}$ Brian J. Rohde, ${ }^{b}$ Megan L. Robertson, $* a$ \\ and Ramanan Krishnamoorti*a
}

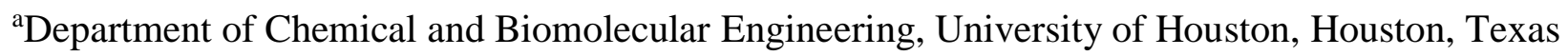
77204, United States

${ }^{\mathrm{b}}$ ExxonMobil Chemical Company, Baytown, Texas 77520, United States

\begin{abstract}
*Corresponding authors
University of Houston

4726 Calhoun Road

S222 Engineering Building 1

Houston, TX 77204-4004

Krishnamoorti: ramanan@uh.edu, Robertson: mlrobertson@uh.edu

Krishnamoorti: 713-743-4307, Robertson: 713-743-2748
\end{abstract}




\section{$\underline{\text { Safety Notes }}$}

Saturation Reactions: Please note the potential dangers present when operating high pressure reactors and using flammable hydrogen and deuterium gases. Only sufficiently trained personnel should operate high pressure reactors.

Anionic Polymerization: Please note the potential dangers present when working with vacuum and pressurized glassware (such as a Schlenk line), using liquid nitrogen to freeze reagents (with the potential to condense liquid oxygen), and when handling pyrophoric chemicals. Only sufficiently trained personnel should conduct anionic polymerizations. 
(a)

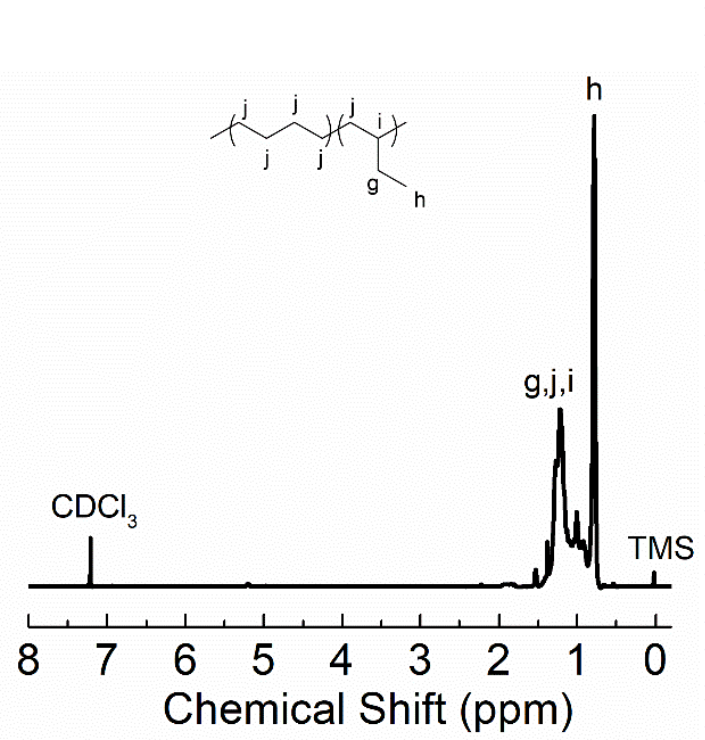

(b)

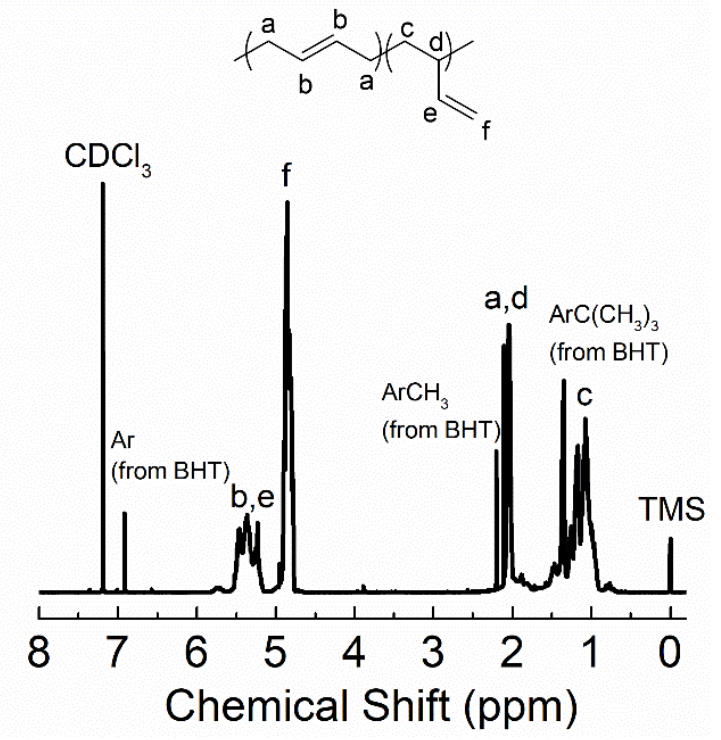

Figure S1: ${ }^{1} \mathrm{H}$ NMR data obtained from a) PEE-20k and b) PBD precursor to PEE-20k (1,4-PBD content was less than 1\%). The disappearance of peaks associated with the 1,2-PBD precursor (peaks c, d, e, and f) indicates saturation level > 99\% in a). 


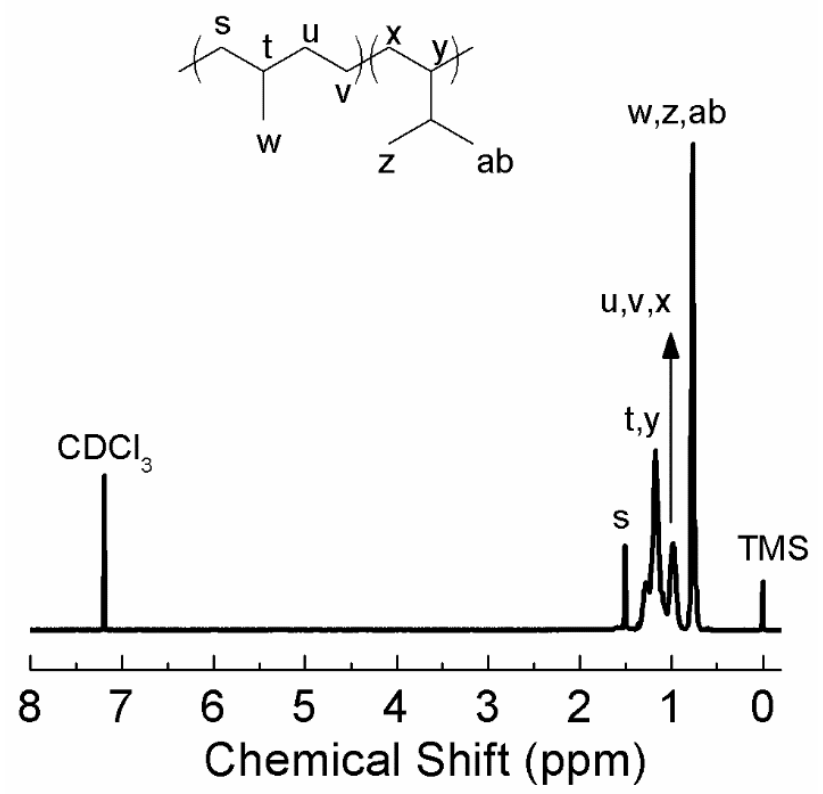

Figure S2: ${ }^{1} \mathrm{H}$ NMR data obtained from PEP-11k (A). The disappearance of peaks associated with the 1,4-PI precursor (shown below in Figure S3) indicates full saturation.

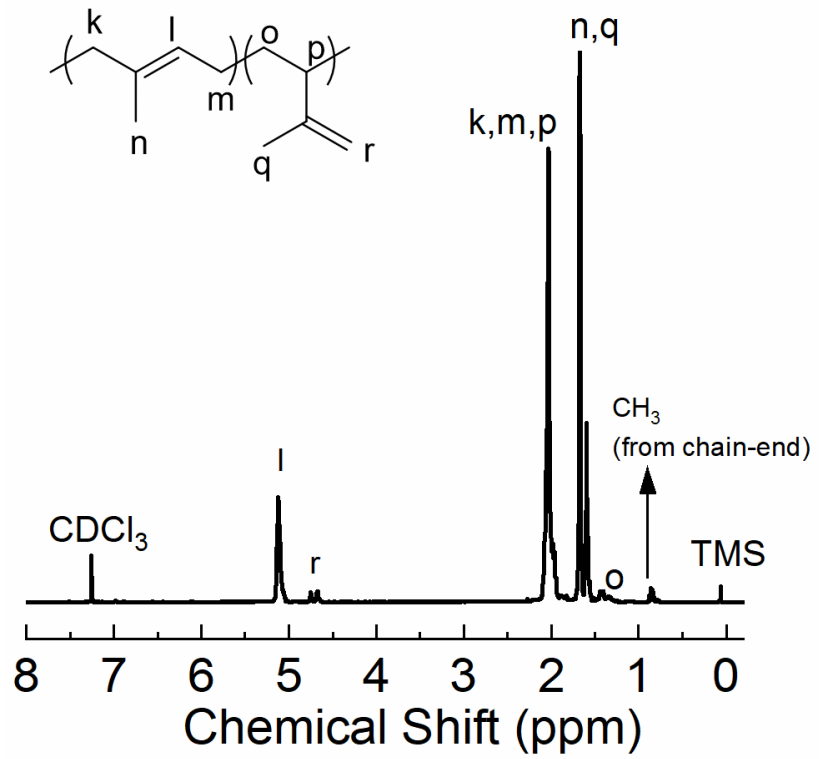

Figure S3: ${ }^{1} \mathrm{H}$ NMR data obtained from PIEP0-5k. 


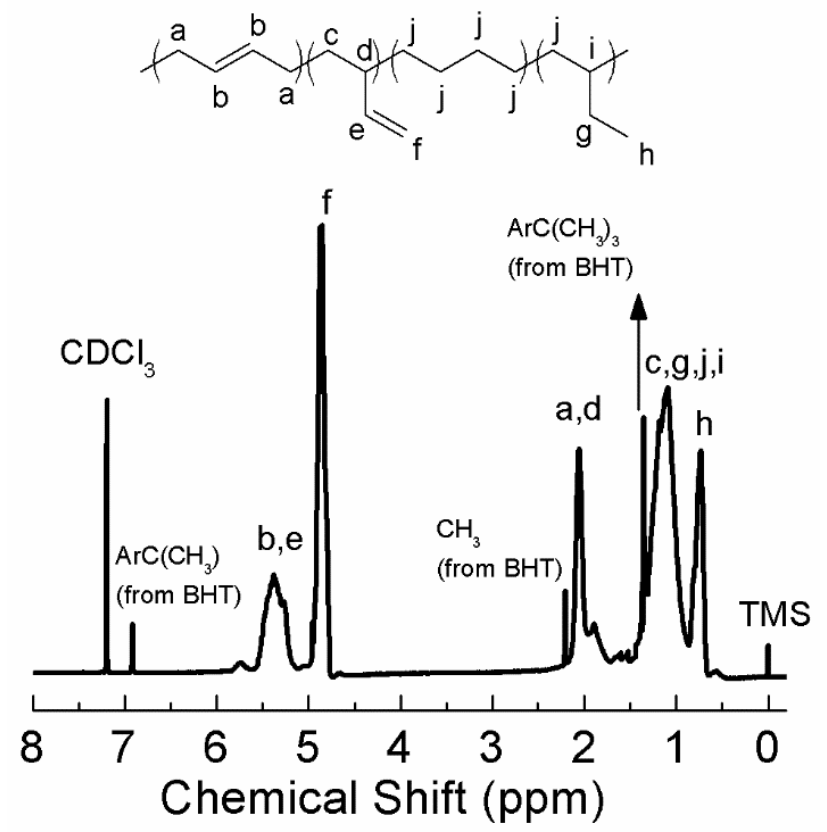

Figure S4: ${ }^{1} \mathrm{H}$ NMR data obtained from PBDEE27-3k.

The mol $\%$ of PEE was quantified using ${ }^{1} \mathrm{H}$ NMR data obtained from 1,2-PBD-co-PEE (representative data set shown in Figure S4):

$\operatorname{mol} \% P E E=\frac{2 h}{2 h+3 f}$

The mol $\%$ of PEE was then converted to vol \% of PEE using the density of PEE and 1,2-PBD from our previous publication. ${ }^{1}$ The presence of 1,4 -addition in the PBD precursor $(<2 \%$ as shown in Table 1 in main text) and its saturated analogue were not considered in the calculation. 


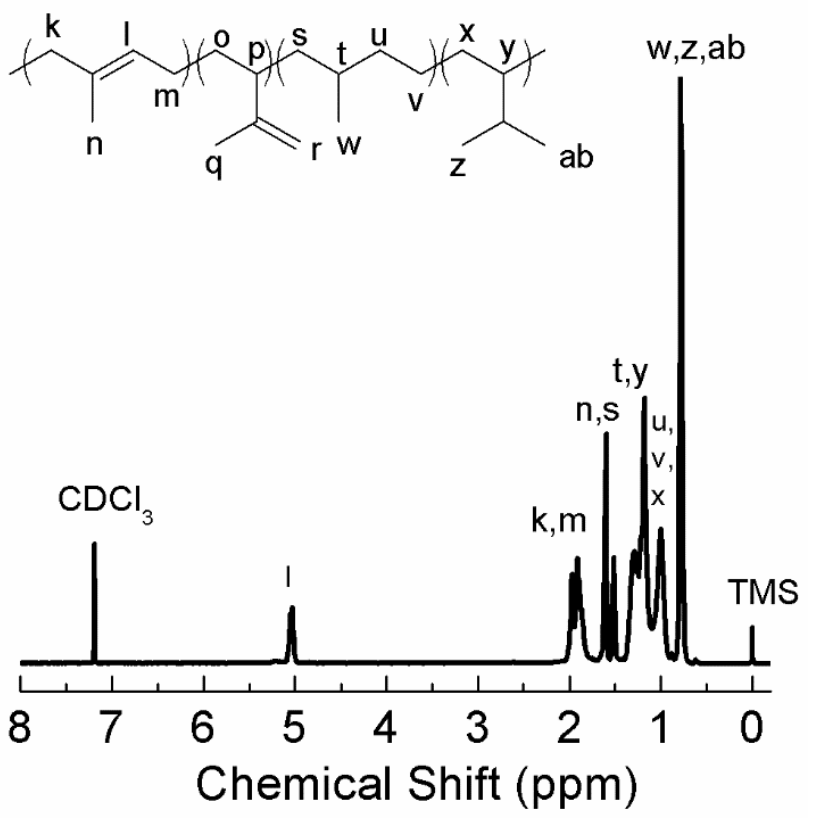

Figure S5: ${ }^{1} \mathrm{H}$ NMR data obtained from PIEP64-24k. The disappearance of peaks associated with 3,4-PI (peaks o, p, q and r) indicates full saturation.

The mol $\%$ of PEP was quantified using ${ }^{1} \mathrm{H}$ NMR data obtained from 1,4-PI-co-PEP copolymer (shown in Figure S5):

$\operatorname{mol} \% P E P=\frac{(n, s)-3 l}{(n, s)-l}$

The mol $\%$ of PEP was then converted to vol \% of PEP using the density of 1,4-PI listed in Table 2 of main text and the density of PEP measured as $0.8509 \mathrm{~g} / \mathrm{cm}^{3}$. The presence of $6 \% 3,4$-additon in the PI precursor (as shown in Table 1 in main text) and its saturated analogue were not considered in the calculation. 


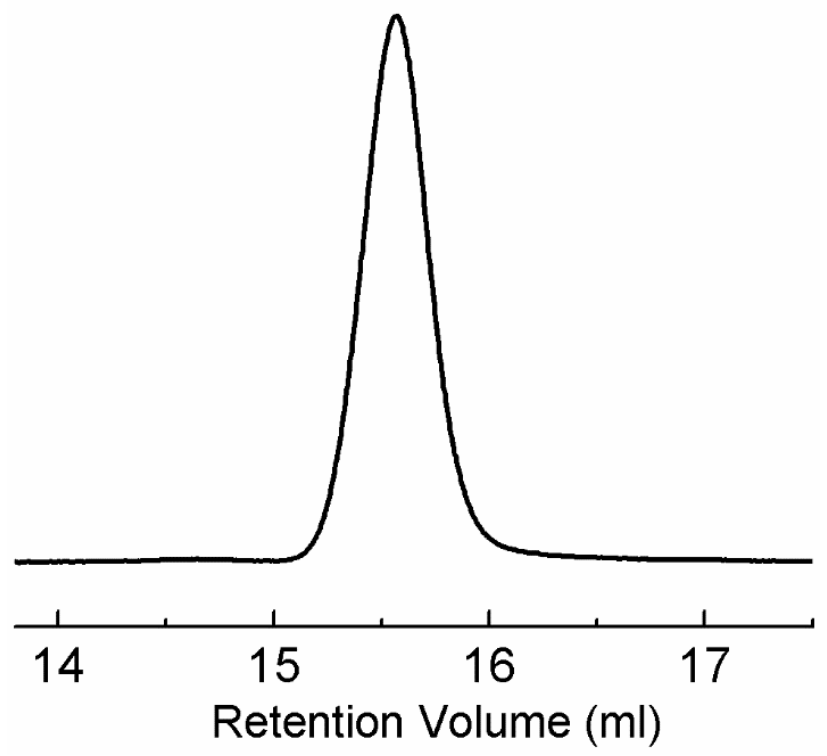

Figure S6: GPC data obtained from PEE-20k.

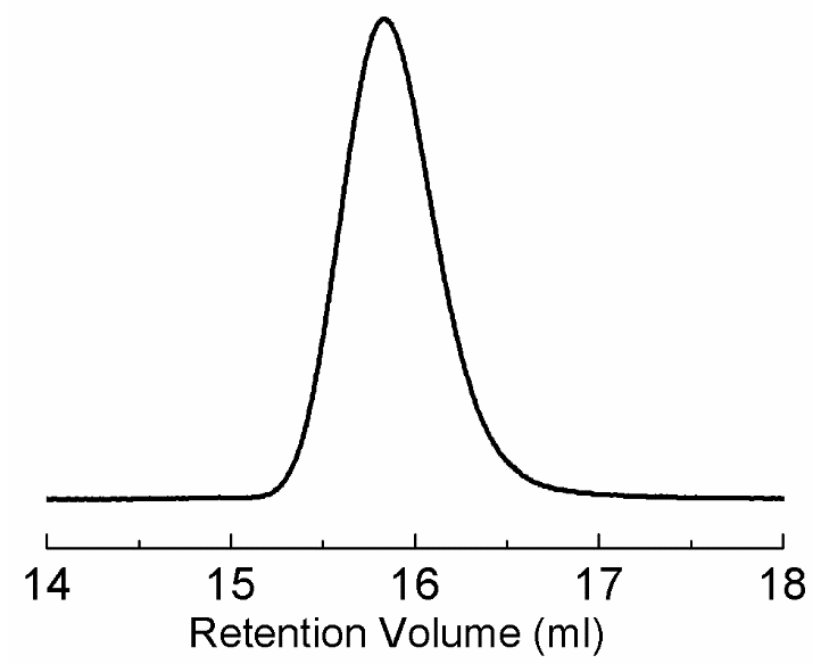

Figure S7: GPC data obtained from PEP-11k (A). 


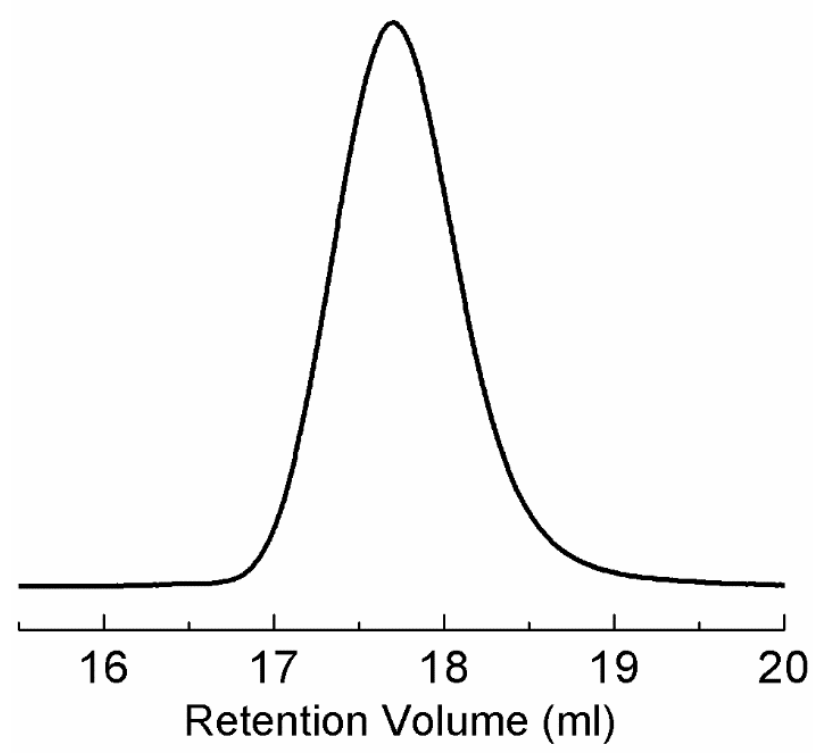

Figure S8: GPC data obtained from PIEP0-5k.

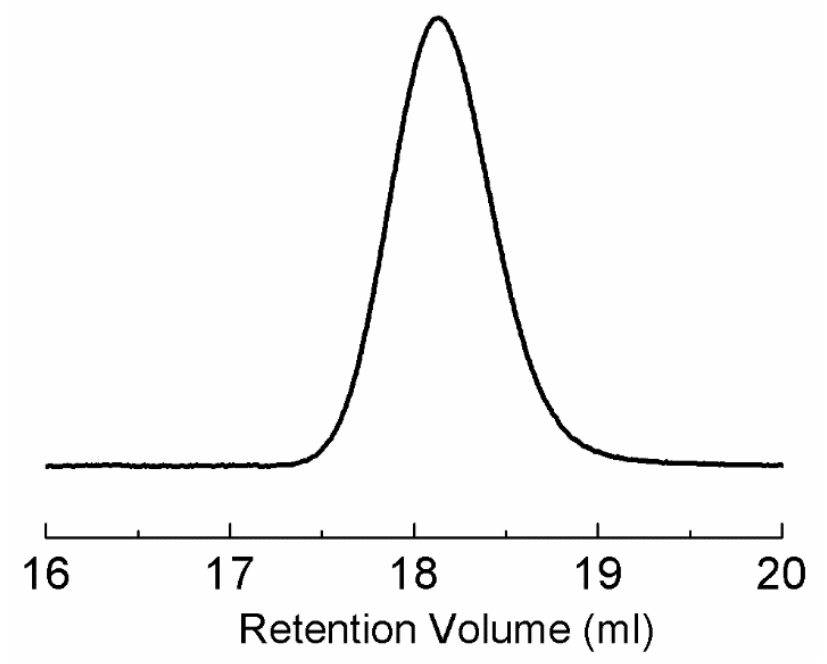

Figure S9: GPC data obtained from PBDEE27-3k. 


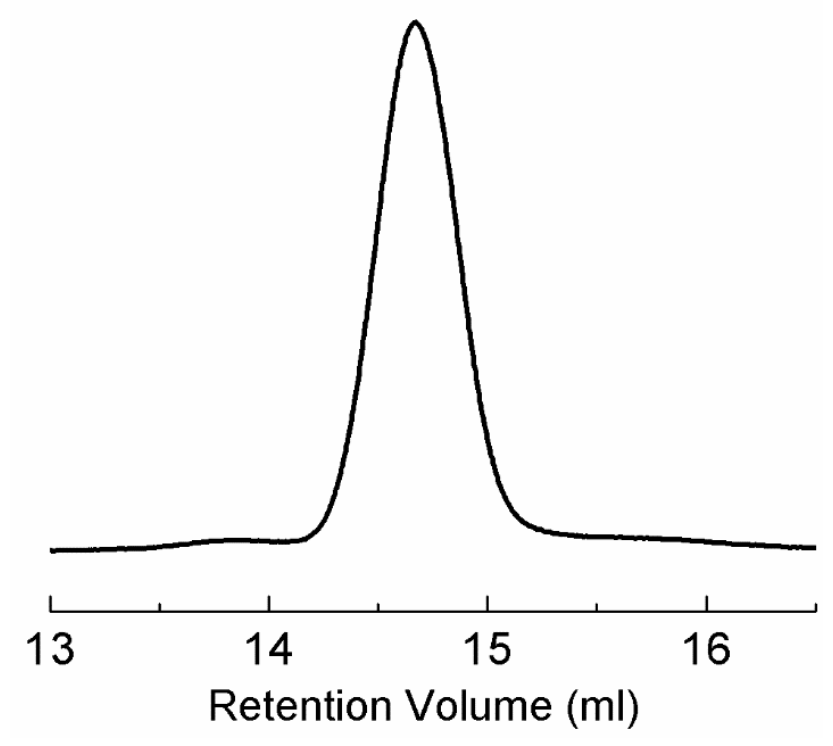

Figure S10: GPC data obtained from PIEP64-24k. 
SANS Data and Analysis for 1,2-PBD-co-PEE / PEE Blends
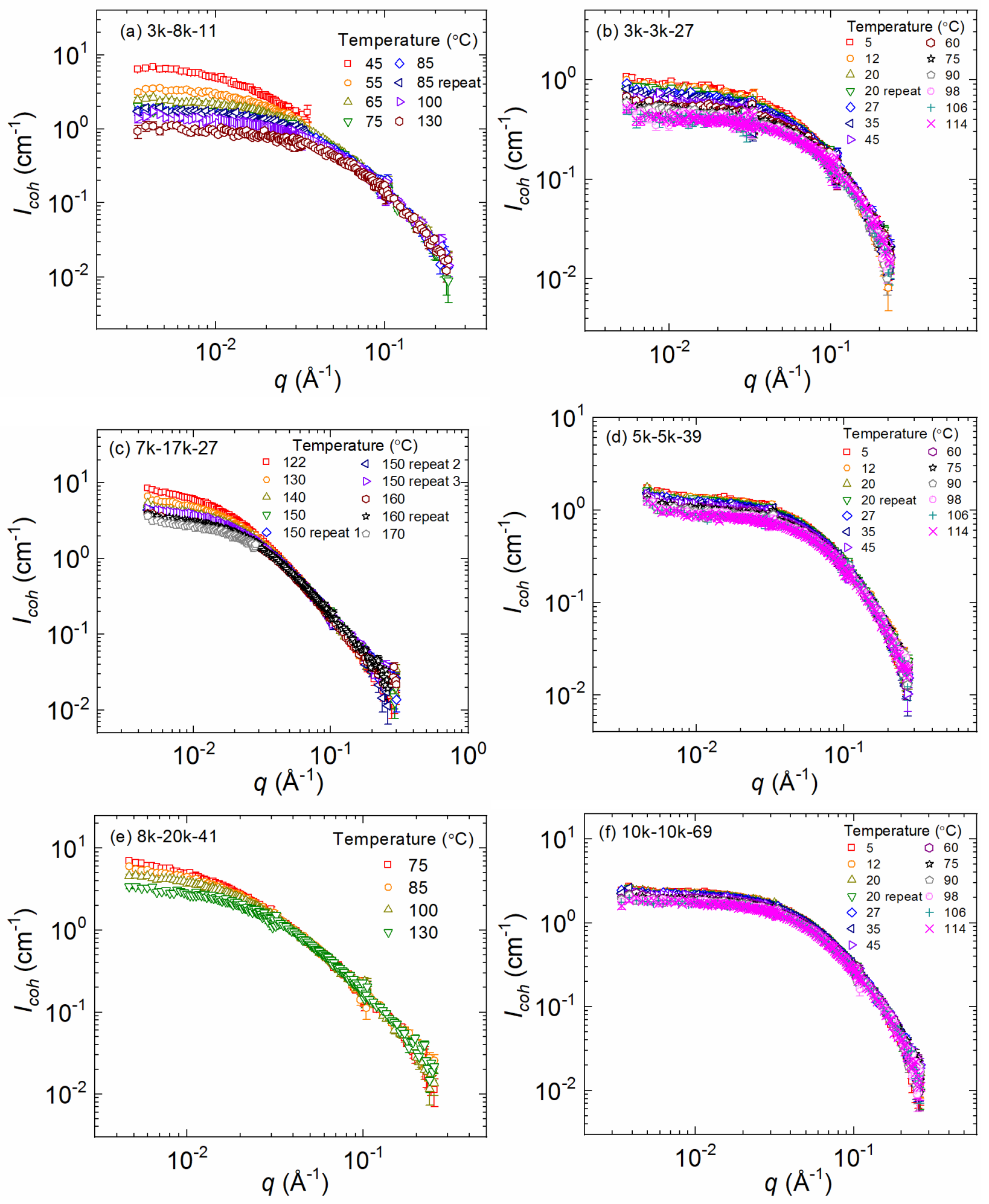

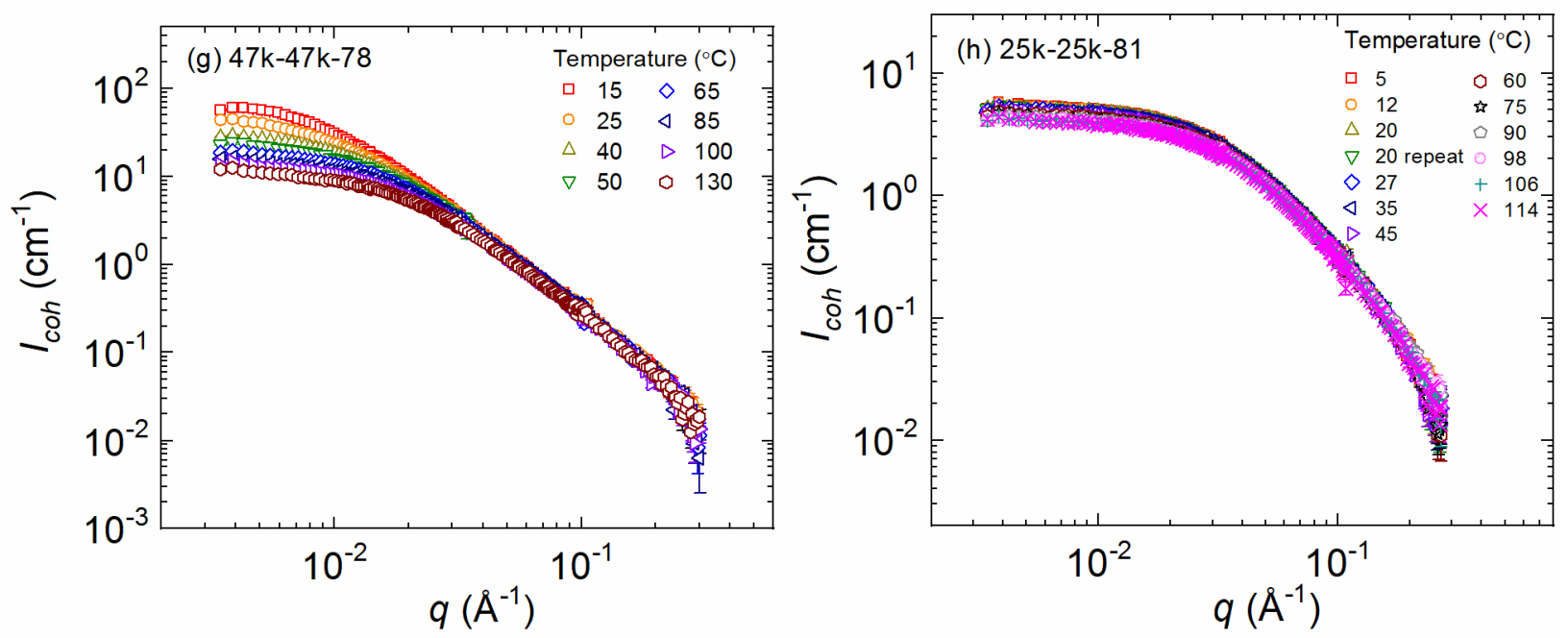

Figure S11: Coherent SANS intensity vs. $q$ obtained for 1,2-PBD-co-PEE / PEE blends. The error bars represent the uncertainty in $I_{c o h}$. In many cases, error bars are smaller than the data points and not shown. 


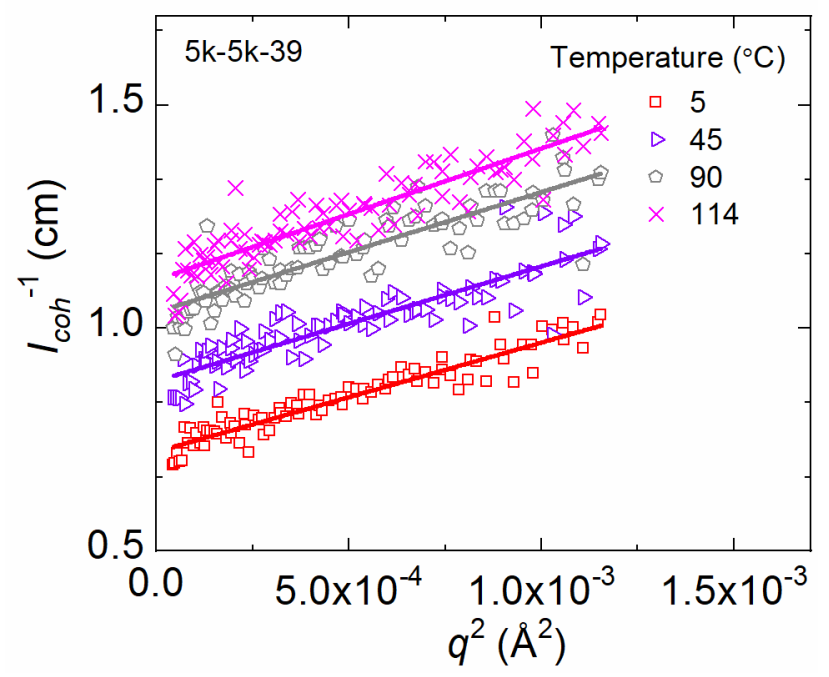

Figure S12: Zimm plot $I_{c o h}{ }^{-1}$ vs. $q$ obtained for blend $5 \mathrm{k}-5 \mathrm{k}-39$ at representative temperatures. 

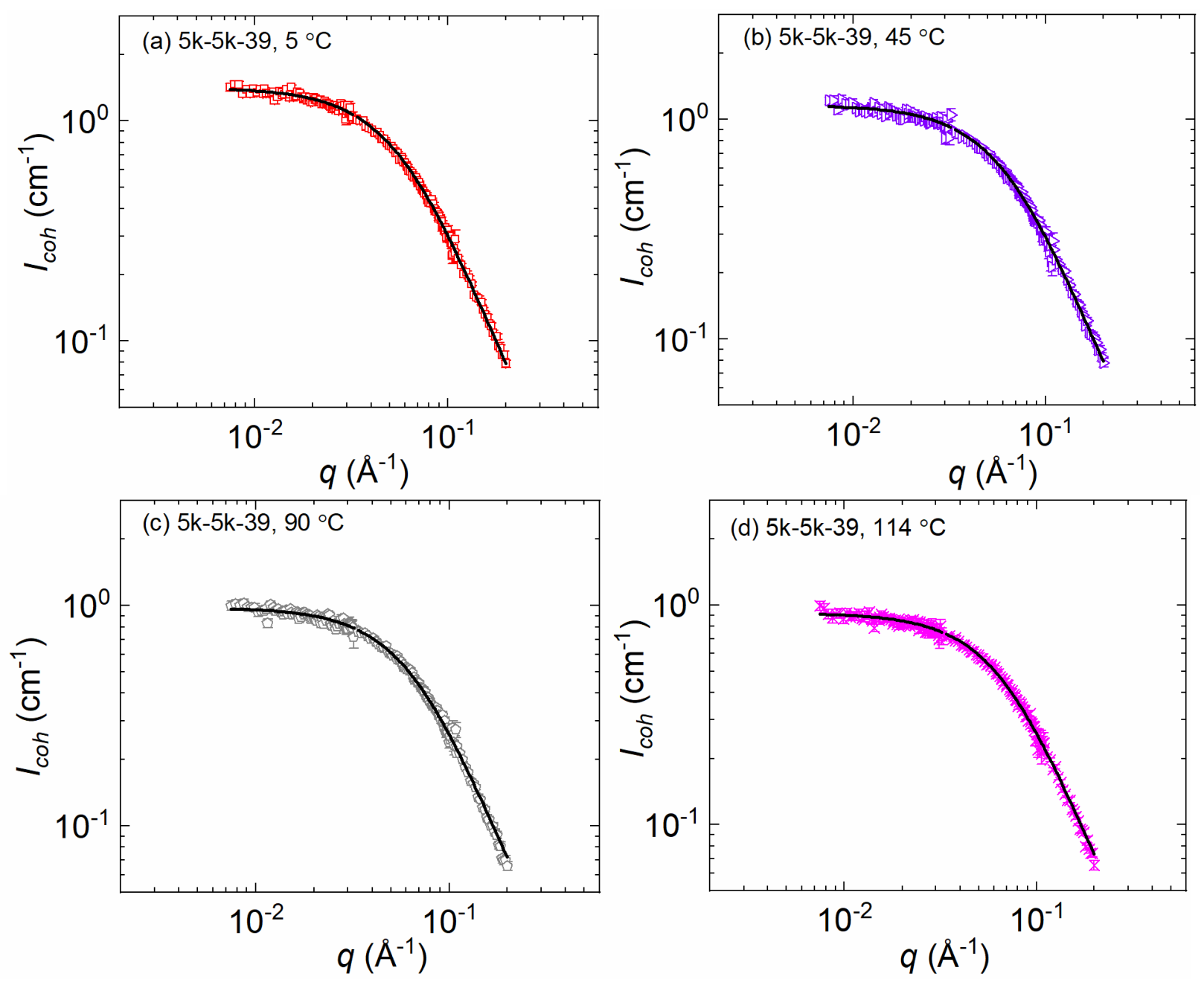

Figure S13: Representative SANS data obtained from blend 5k-5k-39. The Random Phase Approximation was fit to the data. $\chi$ and $\alpha$ were adjustable parameters in the data fitting. The error bars represent the uncertainty in $I_{c o h}$. In many cases, error bars are smaller than the data points and not shown. 
${ }^{13}$ C NMR Data and Analysis

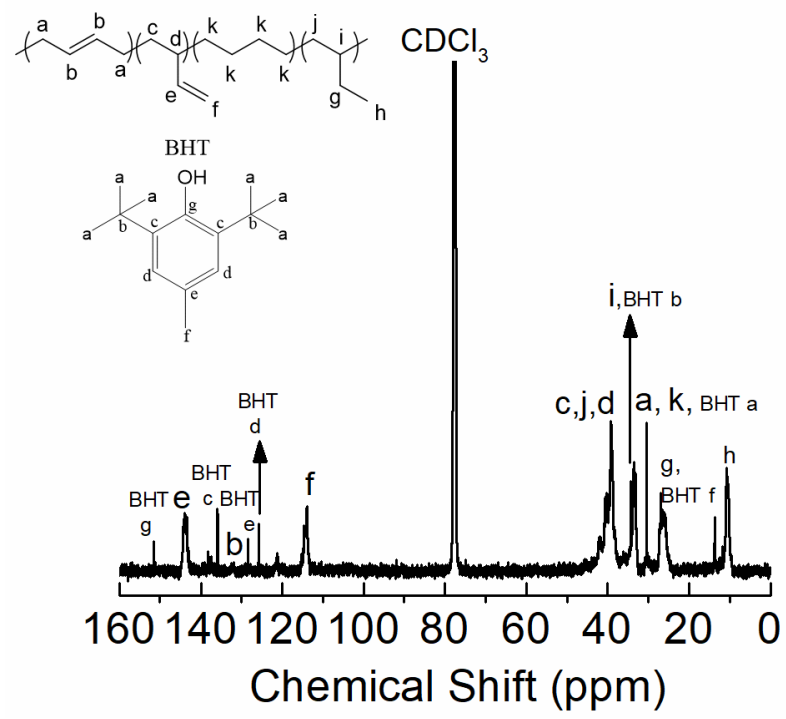

Figure S14: ${ }^{13} \mathrm{C}$ NMR data obtained from PBDEE61-20k.

Table S1: Predicted ${ }^{13} \mathrm{C}$ NMR Chemical Shift (ppm) of Alkane Carbons in the Triad Sequences Composed of Repeat Unit Type A (1,2-PBD) and Repeat Unit Type B (PEE), with Peaks Labeled in Figure S14.

\begin{tabular}{|l|l|l|l|l|l|l|}
\hline & AAA & BBB & BAB & BAA & ABA & BBA \\
\hline c & 39.1 & NA & 41.1 & 41.3 & 38.9 & 41.1 \\
\hline d & 39.1 & NA & 38.9 & 39 & 39 & 38.9 \\
\hline j & NA & 43.8 & 41.1 & 41.3 & 41.3 & 41.1 \\
\hline i & NA & 34.3 & 34.4 & 34.5 & 34.5 & 34.4 \\
\hline g & NA & 26.5 & 26.6 & 26.7 & 26.7 & 26.6 \\
\hline h & NA & 11.9 & 11.9 & 11.9 & 11.9 & 11.9 \\
\hline
\end{tabular}




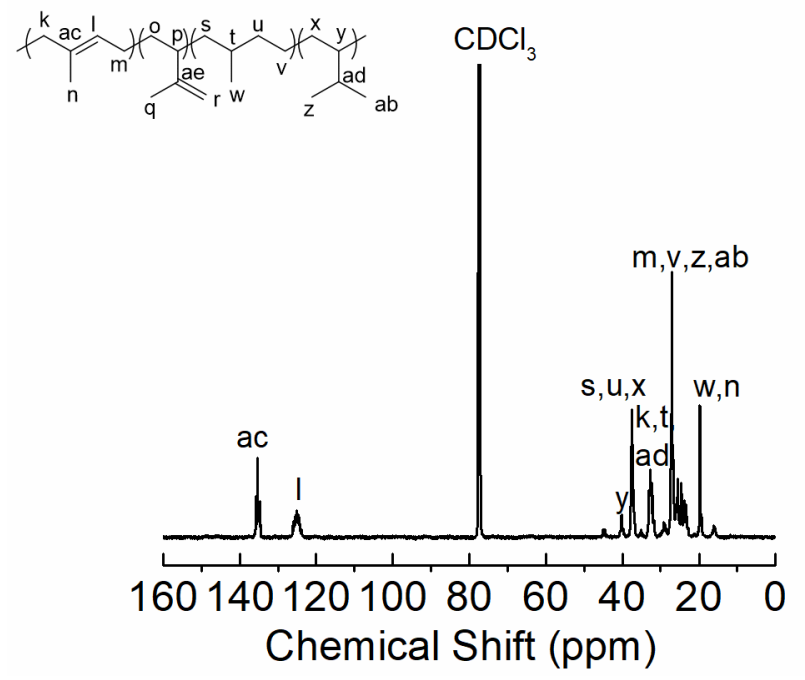

Figure S15: ${ }^{13} \mathrm{C}$ NMR data obtained from PIEP64-24k. The disappearance of peaks from 3,4-PI (peaks $\mathrm{o}, \mathrm{p}, \mathrm{q}, \mathrm{r}$ and ae) indicates full saturation.

Table S2: Predicted ${ }^{13} \mathrm{C}$ NMR Chemical Shift (ppm) of Alkane Carbons in the Triad Sequences Composed of Repeat Unit Type A (1,4-PI) and Repeat Unit Type B (PEP), with Peaks Labeled in Figure S15.

\begin{tabular}{|l|l|l|l|l|l|l|}
\hline & AAA & BBB & BAB & BAA & ABA & BBA \\
\hline $\mathrm{k}$ & 39.7 & NA & 39.6 & 39.6 & 39.7 & 39.6 \\
\hline m & 26.7 & NA & 30.1 & 26.7 & 30.1 & 30.1 \\
\hline n & 16.4 & NA & 16.3 & 16.3 & 16.4 & 16.3 \\
\hline s & NA & 37.7 & 37.3 & 38 & 38 & 38 \\
\hline t & NA & 33.2 & 33.3 & 38.9 & 38.9 & 38.8 \\
\hline u & NA & 37.7 & 37.8 & 37.9 & 37.9 & 37.8 \\
\hline v & NA & 24.6 & 24.9 & 24.9 & 24.9 & 24.6 \\
\hline w & NA & 21 & 21 & 21.1 & 21.1 & 21.1 \\
\hline
\end{tabular}


Tables S1 and S2 show the predicted chemical shifts of alkane carbons observed in ${ }^{13} \mathrm{C}$ NMR data, for triad sequences composed of A and B repeat units (1,2-PBD and PEE repeat units, respectively in 1,2-PBD-co-PEE or 1,4-PI and PEP repeat units, respectively, in 1,4-PI-co-PEP). In order to simplify the analysis, the minor contents of $1,4-\mathrm{PBD}, 3,4-\mathrm{PI}$ and their saturated analogues were neglected. The predicted chemical shifts were calculated using Grant and Paul Empirical Rules as: ${ }^{2}$

$$
\delta(k)=-2.3+\sum_{l} A_{l} n_{k l}+\sum_{j} B_{j}
$$

where $\delta(k)$ is the ${ }^{13} \mathrm{C}$ chemical shift of the $k$ th carbon, -2.3 is the chemical shift in ppm of methane carbon, $n_{k l}$ is the number of carbon atoms in the $l$ th position relative to the $k$ th carbon, and $A_{l}$ is the additive chemical shift assigned to the $l$ th carbon as listed in Table $\mathrm{S} 3$ (a). ${ }^{2-3} B_{j}$ is the corrective term. Quaternary, tertiary, secondary and primary carbons are designated as $4^{\circ}, 3^{\circ}, 2^{\circ}$, and $1^{\circ}$, respectively. In Table S3 (b), the values of $B_{j}$ are tabulated (carbon $k$ classification is indicated first, and the classification of the carbon in the $\alpha$ position is listed in parentheses). ${ }^{2}$ 
Table S3 (a): Coefficients $A_{l}$ Used in Equation S3

\begin{tabular}{|l|l|l|}
\hline $\begin{array}{l}\text { Position of carbon } l \\
\text { relative to carbon } k\end{array}$ & $-\mathrm{C}$ & $-\mathrm{C}=\mathrm{C}$ \\
\hline$\alpha$ & 9.1 & 19.5 \\
\hline$\beta$ & 9.4 & 6.9 \\
\hline$\gamma$ & -2.5 & -2.1 \\
\hline$\delta$ & 0.3 & 0.8 \\
\hline
\end{tabular}

Table S3 (b): Coefficients $B_{j}$ Used in Equation $\mathrm{S3}^{\mathrm{a}}$

\begin{tabular}{|l|l|}
\hline $\begin{array}{l}\text { Carbon } k \text { classification (classification } \\
\text { of carbon in } \alpha \text { position) }\end{array}$ & $B_{j}$ \\
\hline $1^{\circ}\left(3^{\circ}\right)$ & -1.1 \\
\hline $1^{\circ}\left(4^{\circ}\right)$ & -3.4 \\
\hline $2^{\circ}\left(3^{\circ}\right)$ & -2.5 \\
\hline $2^{\circ}\left(4^{\circ}\right)$ & -7.2 \\
\hline $3^{\circ}\left(2^{\circ}\right)$ & -3.7 \\
\hline $3^{\circ}\left(3^{\circ}\right)$ & -9.5 \\
\hline $3^{\circ}\left(4^{\circ}\right)$ & -15.0 \\
\hline $4^{\circ}\left(1^{\circ}\right)$ & -1.5 \\
\hline $4^{\circ}\left(2^{\circ}\right)$ & -8.4 \\
\hline $4^{\circ}\left(3^{\circ}\right)$ & -15.0 \\
\hline $4^{\circ}\left(4^{\circ}\right)$ & -25.0 \\
\hline
\end{tabular}

${ }^{\mathrm{a}}$ If not listed in the table, $B_{j}=0$ 


\section{Comparison of SANS Data Obtained for 1,2-PBD / PEE Blend}

In our previous study, we measured $\chi(\mathrm{T})$ of 1,2-PBD and PEE using two separate blends (at differing molecular weights) in order access a wider temperature range, in one case $5-70{ }^{\circ} \mathrm{C}$ and in the other, $118-166^{\circ} \mathrm{C} .{ }^{1}$ More recently, we repeated these measurements (Figure S16). After acquiring $\chi(\mathrm{T})$ for the blends of 1,2-PBD and PEE with 2 freshly prepared blends (blend characteristics shown in Tables S4 and S5 for the recent measurements) in the $50-170{ }^{\circ} \mathrm{C}$ temperature range, we observed better agreement in the quantified chain dimensions (Figure S17). Moreover, the newly prepared blends from the recent measurement were validated through thermal cycling, i.e., the blends were cycling between heating up and cooling down to across the phase boundaries when acquiring the data. The convergence of the newly acquired data from multiple blends with the old measurement at $5-70{ }^{\circ} \mathrm{C}$ range provided more confidence. Though we can't be certain as to the origin of the discrepancy between the previous study and present study at high temperatures, we suspect that for the prior measurements, the samples were not fully equilibrated. In the recent measurements reported here, thermal cycling confirmed the full equilibration of the sample at low and high temperatures. 


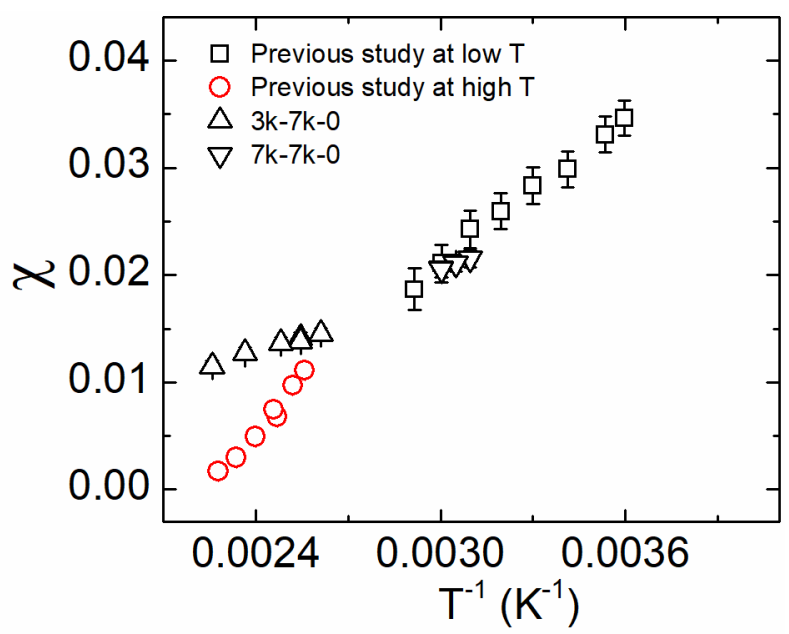

Figure S16: Comparison of $\chi\left(\mathrm{T}^{-1}\right)$ of 1,2-PBD / PEE quantified from three independent measurements: 1 ) previously reported data (ref. ${ }^{1}$ ) obtained on a lower molecular weight blend in the low temperature region (black $\square$ ), 2) previously reported data (ref. ${ }^{1}$ ) obtained on a higher molecular weight blend in the high temperature region (red $\circ$ ), and 3) newly acquired data with two freshly prepared blends (black $\triangle$ and $\nabla$ ) different in molecular weight. Blends characteristics are shown in Tables S4 and S5 for the newly acquired data.

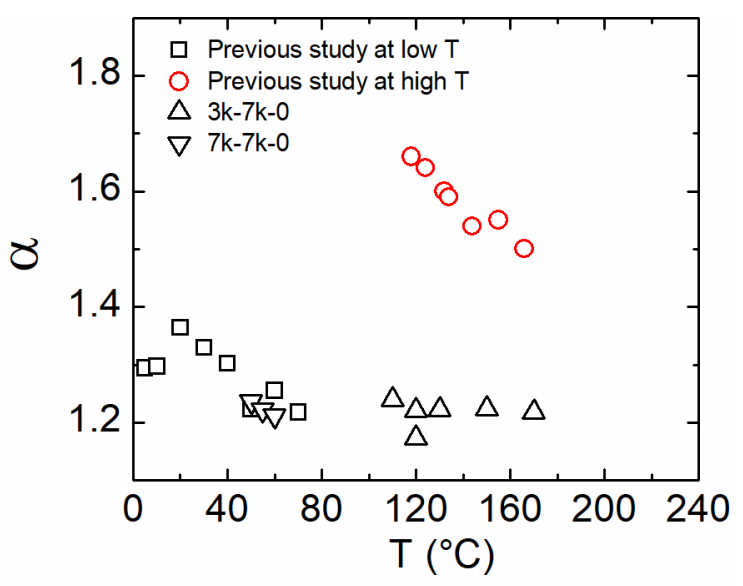

Figure S17: Comparison of chain expansion parameter, $\alpha$, extracted from RPA fitting to SANS data obtained from 1,2-PBD / PEE blends: 1) previously reported data (ref. ${ }^{1}$ ) obtained on a lower molecular weight blend (black $\square$ ), 2) previously reported data (ref. ${ }^{1}$ ) obtained on a higher molecular weight blend (red $\circ$ ), and 3) newly acquired data with two freshly prepared blends (black $\triangle$ and $\nabla$ ) different in molecular weight. $\alpha$ was calculated using literature values of $l_{i}=6.0$ and $4.7 \AA$ for 1,2-PBD and PEE respectively, based on $v_{r e f}=100 \AA^{3 .}{ }^{4}$ 
Table S4: Polymer Characteristics

\begin{tabular}{|l|l|l|l|l|l|l|}
\hline Polymer $^{\mathrm{a}}$ & $\begin{array}{l}\mathrm{M}_{\mathrm{w}} \\
(\mathrm{kg} / \mathrm{mol})^{\mathrm{b}}\end{array}$ & $\mathrm{Ð}^{\mathrm{b}}$ & $\begin{array}{l}\% \quad 1,2 \text { in } \\
\mathrm{PBD}^{\mathrm{c}}\end{array}$ & $\begin{array}{l}\rho(\mathrm{g} / \mathrm{ml}) \\
\text { at } 23{ }^{\circ} \mathrm{C}^{\mathrm{d}}\end{array}$ & $\begin{array}{l}\mathrm{N} \text { at } \\
23{ }^{\circ} \mathrm{C}^{\mathrm{e}}\end{array}$ & $\mathrm{n}_{\mathrm{D}}{ }^{\mathrm{f}}$ \\
\hline PBD-3k & 3.4 & 1.01 & 98.6 & 0.8766 & 63 & $\mathrm{NA}$ \\
\hline PBD-7k & 6.7 & 1.03 & 99.0 & 0.8868 & 124 & NA \\
\hline PEE-7k & 6.7 & 1.03 & 99.0 & 0.9040 & 132 & 2.42 \\
\hline
\end{tabular}

${ }^{a} \mathrm{PBD}-\mathrm{Xk}$ refers to $1,2-\mathrm{PBD}$ with $\mathrm{M}_{\mathrm{w}}$ of $\mathrm{X} \mathrm{kg} / \mathrm{mol}$. PEE-Xk refers to PEE (prepared by satuation of 1,2-PBD with $\mathrm{D}_{2}$ ) with $\mathrm{M}_{\mathrm{w}}$ of $\mathrm{X} \mathrm{kg} / \mathrm{mol}$.

${ }^{\mathrm{b}}$ Measured for the 1,2-PBD, using GPC with light scattering.

${ }^{\mathrm{c}} \% 1,2$-addition of 1,2-PBD. Measured through ${ }^{1} \mathrm{H}$ NMR, error estimated at $\pm 0.4-0.9$.

${ }^{\mathrm{d}}$ Measured using density gradient column. Error was quantified from $\pm 0.0001-0.0008$.

${ }^{\mathrm{e}}$ Number of repeat units per chain at $23{ }^{\circ} \mathrm{C}$ based on reference volume of $100 \AA^{3}$. Thermal expansion coefficient of $0.00068 \mathrm{~K}^{-1}$ was used to account for temperature dependence of the molar volume..$^{5-6}$

${ }^{\mathrm{f}}$ Number of deuterium atoms per repeat unit was calculated following eq. 2 in main text.

Table S5: 1,2-PBD / PEE Blend Characteristics

\begin{tabular}{|l|l|l|l|l|}
\hline Blend & Component 1: 1,2-PBD & Component 2: PEE $^{\mathrm{b}}$ & $\phi_{1}{ }^{\mathrm{a}}$ & $\begin{array}{l}\mathrm{T}_{\mathrm{c}, \text { SANS }^{\mathrm{d}}} \\
\left({ }^{\circ} \mathrm{C}\right)\end{array}$ \\
\hline 3k-7k-0 & PBD-3k & PEE-7k & 0.590 & $<50$ \\
\hline $7 \mathrm{k}-7 \mathrm{k}-0$ & PBD-7k & PEE-7k & 0.498 & $<110$ \\
\hline
\end{tabular}

${ }^{a}$ Obtained through anionic polymerization of 1,3-butadiene with DIPIP as polar additive.

${ }^{\mathrm{b}}$ Obtained through saturation of 1,2-PBD with $\mathrm{D}_{2}$.

${ }^{\mathrm{c}}$ Blend compositions were near the critical compositions for all blends.

${ }^{\mathrm{d}}$ Critical temperature quantified through SANS. 


\section{Summary of $\chi$ and $\alpha$ Parameters for 1,2-PBD-co-PEE / PEE Blends}

Table S6: $\chi$ extracted through Zimm Analysis and RPA Fitting and $\alpha$ Extracted from RPA Fitting

(a) Blend $3 \mathrm{k}-8 \mathrm{k}-11$

\begin{tabular}{|l|l|l|l|l|l|l|l|l|}
\hline $\mathrm{T}\left({ }^{\circ} \mathrm{C}\right)$ & 45 & 55 & 65 & 75 & 85 & 85 repeat & 100 & 130 \\
\hline$\chi_{\text {Zimm }^{\mathrm{a}}}$ & $0.0202 \pm$ & $0.0189 \pm$ & $0.0176 \pm$ & $0.0161 \pm$ & $0.0159 \pm$ & $0.0161 \pm$ & $0.0148 \pm$ & $0.0123 \pm$ \\
& 0.0008 & 0.0008 & 0.0008 & 0.0008 & 0.0008 & 0.0008 & 0.0008 & 0.0008 \\
\hline$\chi_{\mathrm{RPA}^{\mathrm{b}}}$ & NA & NA & $0.0174 \pm$ & $0.0160 \pm$ & $0.0158 \pm 0$ & NA & $0.0147 \pm$ & $0.0122 \pm$ \\
& & & 0.0012 & 0.0011 & .0011 & & 0.0013 & 0.0011 \\
\hline$\alpha^{\mathrm{c}}$ & NA & NA & 1.16 & 1.18 & 1.19 & NA & 1.18 & 1.15 \\
\hline
\end{tabular}

(b) Blend $3 \mathrm{k}-3 \mathrm{k}-27$

\begin{tabular}{|l|l|l|l|l|l|l|l|l|}
\hline $\mathrm{T}\left({ }^{\circ} \mathrm{C}\right)$ & 5 & 12 & 20 & 20 repeat & 27 & 35 & 45 & 60 \\
& & & & & & & & \\
\hline$\chi \mathrm{Zimm}^{\mathrm{a}}$ & $0.0195 \pm$ & $0.0185 \pm$ & $0.0173 \pm$ & $0.0172 \pm$ & $0.0164 \pm$ & $0.0148 \pm$ & $0.0134 \pm$ & $0.0117 \pm$ \\
& 0.0014 & 0.0014 & 0.0015 & 0.0015 & 0.0015 & 0.0016 & 0.0016 & 0.0017 \\
\hline$\chi_{\mathrm{RPA}^{\mathrm{b}}}$ & $0.0187 \pm 0$ & $0.0179 \pm$ & $0.0169 \pm$ & $0.0162 \pm 0$. & $0.0152 \pm$ & $0.0140 \pm$ & $0.0129 \pm$ & $0.0110 \pm$ \\
& .0011 & 0.0011 & 0.0010 & 0010 & 0.0009 & 0.0008 & 0.0008 & 0.0007 \\
\hline$\alpha^{\mathrm{c}}$ & 1.13 & 1.15 & 1.16 & 1.11 & 1.13 & 1.13 & 1.13 & 1.13 \\
\hline
\end{tabular}

\begin{tabular}{|l|l|l|l|l|l|}
\hline $\mathrm{T}\left({ }^{\circ} \mathrm{C}\right)$ & 75 & 90 & 98 & 106 & 114 \\
\hline$\chi_{\mathrm{Zimm}^{\mathrm{a}}}$ & $0.0103 \pm 0.0018$ & $0.0089 \pm 0.0018$ & $0.0074 \pm 0.0019$ & $0.0071 \pm 0.0019$ & $0.0067 \pm 0.0019$ \\
\hline$\chi_{\mathrm{RPA}^{\mathrm{b}}}$ & $0.0093 \pm 0.0006$ & $0.0078 \pm 0.0005$ & $0.0069 \pm 0.0005$ & $0.0061 \pm 0.0004$ & $0.0052 \pm 0.0005$ \\
\hline$\alpha^{\mathrm{c}}$ & 1.10 & 1.10 & 1.09 & 1.11 & 1.10 \\
\hline
\end{tabular}

(c) $\quad$ Blend $7 \mathrm{k}-17 \mathrm{k}-27$

\begin{tabular}{|c|c|c|c|c|c|c|c|c|}
\hline $\mathrm{T}\left({ }^{\circ} \mathrm{C}\right)$ & 122 & 130 & 140 & 150 & 150 repeat & $\begin{array}{l}150 \\
\text { repeat } 2\end{array}$ & $\begin{array}{l}150 \\
\text { repeat } 3\end{array}$ & 160 \\
\hline$\chi_{\text {Zimm }^{a}}{ }^{a}$ & $\begin{array}{l}0.0085 \pm \\
0.0004\end{array}$ & $\begin{array}{l}0.0081 \pm \\
0.0004\end{array}$ & $\begin{array}{l}0.0076 \pm \\
0.0004\end{array}$ & $\begin{array}{l}0.0071 \pm \\
0.0004\end{array}$ & $\begin{array}{l}0.0071 \pm \\
0.0004\end{array}$ & $\begin{array}{l}0.0073 \pm \\
0.0004\end{array}$ & $\begin{array}{l}0.0074 \pm \\
0.0004\end{array}$ & $\begin{array}{l}0.0068 \pm \\
0.0004\end{array}$ \\
\hline$\chi_{\mathrm{RPA}^{b}}$ & $\begin{array}{l}0.0083 \pm 0 \\
.0007\end{array}$ & $\begin{array}{l}0.0079 \pm \\
0.0007\end{array}$ & $\begin{array}{l}0.0075 \pm \\
0.0007\end{array}$ & $\begin{array}{l}0.0069 \pm \\
0.0006\end{array}$ & $\begin{array}{l}0.0069 \pm 0.0 \\
006\end{array}$ & $\begin{array}{l}0.0071 \pm \\
0.0006\end{array}$ & $\begin{array}{l}0.0071 \pm \\
0.0006\end{array}$ & $\begin{array}{l}0.0068 \pm \\
0.0006\end{array}$ \\
\hline$\alpha^{\mathrm{c}}$ & 1.18 & 1.17 & 1.16 & 1.16 & 1.15 & 1.17 & 1.16 & 1.16 \\
\hline
\end{tabular}

\begin{tabular}{|l|l|l|}
\hline $\mathrm{T}\left({ }^{\circ} \mathrm{C}\right)$ & 160 & 170 \\
\hline$\chi_{\mathrm{Zimm}^{\mathrm{a}}}{ }^{\mathrm{a}}$ & $0.0069 \pm 0.0004$ & $0.0064 \pm 0.0004$ \\
\hline$\chi_{\mathrm{RPA}}{ }^{\mathrm{b}}$ & $0.00068 \pm 0.0006$ & NA \\
\hline$\alpha^{\mathrm{c}}$ & 0.0068 & NA \\
\hline
\end{tabular}


(d) Blend 5k-5k-39

\begin{tabular}{|l|l|l|l|l|l|l|l|l|}
\hline $\begin{array}{l}\mathrm{T} \\
\left({ }^{\circ} \mathrm{C}\right)\end{array}$ & 5 & 12 & 20 & 20 repeat & 27 & 35 & 45 & 60 \\
\hline$\chi_{\text {Zimm }^{\mathrm{a}}}$ & $0.0116 \pm$ & $0.0113 \pm$ & $0.0109 \pm$ & $0.0108 \pm$ & $0.0105 \pm$ & $0.0099 \pm$ & $0.0096 \pm$ & $0.0091 \pm$ \\
& 0.0008 & 0.0008 & 0.0008 & 0.0008 & 0.0008 & 0.0008 & 0.0008 & 0.0008 \\
\hline$\chi_{\mathrm{RPA}^{\mathrm{b}}}$ & $0.0114 \pm$ & $0.0112 \pm$ & $0.0106 \pm$ & $0.0105 \pm 0$. & $0.0102 \pm$ & $0.0096 \pm$ & $0.0095 \pm$ & $0.0089 \pm$ \\
& 0.0008 & 0.0008 & 0.0007 & 0007 & 0.0007 & 0.0007 & 0.0007 & 0.0006 \\
\hline$\alpha^{\mathrm{c}}$ & 0.95 & 0.95 & 0.94 & 0.97 & 0.94 & 0.94 & 0.93 & 0.93 \\
\hline
\end{tabular}

\begin{tabular}{|l|l|l|l|l|l|}
\hline $\begin{array}{l}\mathrm{T} \\
\left({ }^{\circ} \mathrm{C}\right)\end{array}$ & 75 & 90 & 98 & 106 & 114 \\
\hline$\chi_{\mathrm{Zimm}}{ }^{\mathrm{a}}$ & $0.0084 \pm 0.0008$ & $0.0079 \pm 0.0008$ & $0.0076 \pm 0.0008$ & $0.0074 \pm 0.0008$ & $0.0072 \pm 0.0008$ \\
\hline$\chi_{\mathrm{RPA}^{\mathrm{b}}}$ & $0.0082 \pm 0.0006$ & $0.0076 \pm 0.0005$ & $0.0075 \pm 0.0005$ & $0.0072 \pm 0.0005$ & $0.0069 \pm 0.0005$ \\
\hline$\alpha^{\mathrm{c}}$ & 0.96 & 0.96 & 0.94 & 0.94 & 0.94 \\
\hline
\end{tabular}

(e) Blend $8 \mathrm{k}-20 \mathrm{k}-41$

\begin{tabular}{|l|l|l|l|l|}
\hline $\mathrm{T}\left({ }^{\circ} \mathrm{C}\right)$ & 75 & 85 & 100 & 130 \\
\hline$\chi_{\mathrm{Zimm}^{\mathrm{a}}}$ & $0.0071 \pm 0.0003$ & $0.0068 \pm 0.0003$ & $0.0064 \pm 0.0003$ & $0.0053 \pm 0.0003$ \\
\hline$\chi_{\mathrm{RPA}^{\mathrm{b}}}$ & $0.0068 \pm 0.0005$ & $0.0065 \pm 0.0005$ & $0.0062 \pm 0.0004$ & $0.0051 \pm 0.0004$ \\
\hline$\alpha^{\mathrm{c}}$ & 1.33 & 1.30 & 1.28 & 1.21 \\
\hline
\end{tabular}

(f) Blend 20k-20k-61

\begin{tabular}{|l|l|l|l|l|l|l|l|}
\hline $\mathrm{T}\left({ }^{\circ} \mathrm{C}\right)$ & 25 & 40 & 50 & 65 & 85 & 100 & 130 \\
\hline$\chi_{\mathrm{Zimm}^{\mathrm{a}}}$ & $0.0050 \pm$ & $0.0045 \pm$ & $0.0042 \pm$ & $0.0036 \pm$ & $0.0031 \pm$ & $0.0027 \pm$ & $0.0023 \pm$ \\
& 0.0002 & 0.0002 & 0.0002 & 0.0002 & 0.0002 & 0.0002 & 0.0002 \\
\hline$\chi_{\mathrm{RPA}}{ }^{\mathrm{b}}$ & $0.0050 \pm$ & NA & NA & $0.0036 \pm$ & $0.0031 \pm$ & $0.0027 \pm$ & $0.0022 \pm$ \\
& 0.0004 & & & 0.0003 & 0.0002 & 0.0002 & 0.0002 \\
\hline$\alpha^{\mathrm{c}}$ & 1.21 & NA & NA & 1.20 & 1.20 & 1.17 & 1.13 \\
\hline
\end{tabular}

(g) Blend 10k-10k-69

\begin{tabular}{|l|l|l|l|l|l|l|l|l|}
\hline $\mathrm{T}\left({ }^{\circ} \mathrm{C}\right)$ & 5 & 12 & 20 & 20 repeat & 27 & 35 & 45 & 60 \\
\hline$\chi \mathrm{Zimm}^{\mathrm{a}}$ & $0.0034 \pm$ & $0.0032 \pm$ & $0.0030 \pm$ & $0.0030 \pm$ & $0.0030 \pm$ & $0.0028 \pm$ & $0.0028 \pm$ & $0.0024 \pm$ \\
& 0.0004 & 0.0004 & 0.0004 & 0.0004 & 0.0004 & 0.0004 & 0.0004 & 0.0004 \\
\hline$\chi_{\mathrm{RPA}}{ }^{\mathrm{b}}$ & $0.0034 \pm$ & $0.0032 \pm$ & $0.0030 \pm$ & $0.0030 \pm 0$. & $0.0030 \pm$ & $0.0028 \pm$ & $0.0028 \pm$ & $0.0023 \pm$ \\
& 0.0002 & 0.0002 & 0.0002 & 0002 & 0.0002 & 0.0002 & 0.0002 & 0.0002 \\
\hline$\alpha^{\mathrm{c}}$ & 1.04 & 1.04 & 1.04 & 1.02 & 1.04 & 1.01 & 1.00 & 1.03 \\
\hline
\end{tabular}




\begin{tabular}{|l|l|l|l|l|l|}
\hline $\mathrm{T}\left({ }^{\circ} \mathrm{C}\right)$ & 75 & 90 & 98 & 106 & 114 \\
& & & & & \\
\hline$\chi_{\mathrm{Zimm}^{\mathrm{a}}}$ & $0.0022 \pm$ & $0.0019 \pm$ & $0.0018 \pm$ & $0.0017 \pm$ & $0.0018 \pm$ \\
& 0.0004 & 0.0004 & 0.0004 & 0.0004 & 0.0004 \\
\hline$\chi_{\mathrm{RPA}^{\mathrm{b}}}$ & $0.0022 \pm$ & $0.0019 \pm$ & $0.0018 \pm$ & $0.0017 \pm$ & $0.0017 \pm$ \\
& 0.0002 & 0.0002 & 0.0001 & 0.0001 & 0.0001 \\
\hline$\alpha^{\mathrm{c}}$ & 1.02 & 1.02 & 1.03 & 1.02 & 1.04 \\
\hline
\end{tabular}

(h) $\quad$ Blend 47k-47k-78

\begin{tabular}{|l|l|l|l|l|l|l|l|l|}
\hline $\mathrm{T}\left({ }^{\circ} \mathrm{C}\right)$ & 15 & 25 & 40 & 50 & 65 & 85 & 100 & 130 \\
\hline$\chi_{\mathrm{Zimm}^{\mathrm{a}}}$ & $0.0020 \pm$ & $0.0019 \pm$ & $0.0017 \pm$ & $0.0015 \pm$ & $0.0013 \pm$ & $0.0011 \pm$ & $0.0010 \pm$ & $0.0008 \pm$ \\
& 0.0001 & 0.0001 & 0.0001 & 0.0001 & 0.0001 & 0.0001 & 0.0001 & 0.0001 \\
\hline$\chi_{\mathrm{RPA}}{ }^{\mathrm{b}}$ & $0.0020 \pm$ & $0.0019 \pm$ & NA & NA & $0.0013 \pm$ & $0.0011 \pm$ & $0.0010 \pm$ & $0.0008 \pm$ \\
& 0.0002 & 0.0002 & & & 0.0001 & 0.0001 & 0.0001 & 0.0001 \\
\hline$\alpha^{\mathrm{c}}$ & 1.15 & 1.14 & NA & NA & 1.12 & 1.12 & 1.12 & 1.14 \\
\hline
\end{tabular}

(i) Blend $25 \mathrm{k}-25 \mathrm{k}-81$

\begin{tabular}{|l|l|l|l|l|l|l|l|l|}
\hline $\mathrm{T}\left({ }^{\circ} \mathrm{C}\right)$ & 5 & 12 & 20 & 20 repeat & 27 & 35 & 45 & 60 \\
\hline$\chi_{\mathrm{Zimm}^{\mathrm{a}}}$ & $0.0010 \pm$ & $0.0010 \pm$ & $0.0009 \pm$ & $0.0009 \pm$ & $0.0008 \pm$ & $0.0008 \pm$ & $0.0007 \pm$ & $0.0006 \pm$ \\
& 0.0002 & 0.0002 & 0.0002 & 0.0002 & 0.0002 & 0.0002 & 0.0002 & 0.0002 \\
\hline$\chi_{\mathrm{RPA}^{\mathrm{b}}}$ & $0.0010 \pm$ & $0.0010 \pm$ & $0.0009 \pm$ & $0.0009 \pm 0$. & $0.0008 \pm$ & $0.0008 \pm$ & $0.0007 \pm$ & $0.0006 \pm$ \\
& 0.0001 & 0.0001 & 0.0001 & 0001 & 0.0001 & 0.0001 & 0.0001 & 0.0001 \\
\hline$\alpha^{\mathrm{c}}$ & 1.02 & 1.02 & 1.02 & 1.01 & 1.03 & 1.02 & 1.01 & 1.03 \\
\hline
\end{tabular}

\begin{tabular}{|l|l|l|l|l|l|}
\hline $\mathrm{T}\left({ }^{\circ} \mathrm{C}\right)$ & 75 & 90 & 98 & 106 & 114 \\
\hline$\chi$ Zimm $^{\mathrm{a}}$ & $0.0005 \pm$ & $0.0004 \pm$ & $0.0004 \pm$ & $0.0004 \pm$ & $0.0003 \pm$ \\
& 0.0002 & 0.0002 & 0.0002 & 0.0002 & 0.0002 \\
\hline$\chi_{\mathrm{RPA}}{ }^{\mathrm{b}}$ & $0.0005 \pm$ & $0.0004 \pm$ & $0.0004 \pm$ & $0.0004 \pm$ & $0.0003 \pm$ \\
& 0.0001 & 0.0001 & 0.0001 & 0.0001 & 0.0001 \\
\hline$\alpha^{\mathrm{c}}$ & 1.02 & 1.03 & 1.02 & 1.03 & 1.02 \\
\hline
\end{tabular}

${ }^{a}$ Error represents the uncertainty in $\chi$, calculated by propagating error sources together as described in main text.

${ }^{\mathrm{b}}$ For some temperatures, the SANS data were only acquired at low- $q$ configuration so that $q$ range is not sufficient to quantify the chain dimenssions through RPA fitting, and NA was labeled. Error represents the uncertainty in $\chi$, calculated by propagating error sources together as described in main text.

${ }^{\mathrm{c}}$ The chain expansion factor, $\alpha$, obtained from RPA was calculated using literature values of $l_{i}=$ 6.0 and 4.7 for 1,2-PBD and PEE, respectively, based on $v_{r e f}=100 \AA^{3}$. For some temperatures where only low- $q$ configuration data were acquired and not sufficient for RPA model fitting to extract $\alpha$, NA was labled. 
SANS Data and Analysis for 1,4-PI-co-PEP / PEP Blends
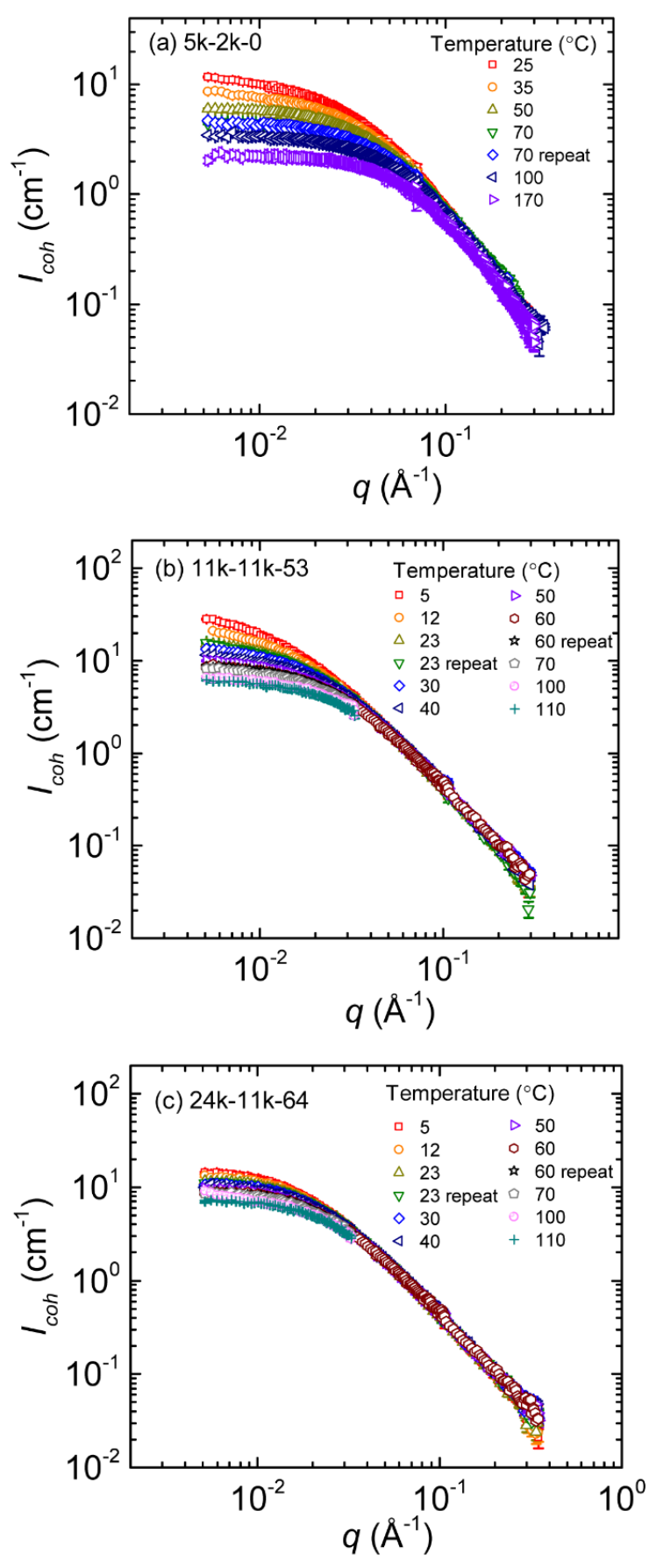

Figure S18: Coherent SANS intensity vs. $q$ obtained for blends containing (a): 1,4-PI / PEP and (b) (c): 1,4-PI-co-PEP / PEP. The error bars represent the uncertainty in $I_{c o h}$. In many cases, error bars are smaller than the data points and not shown. 


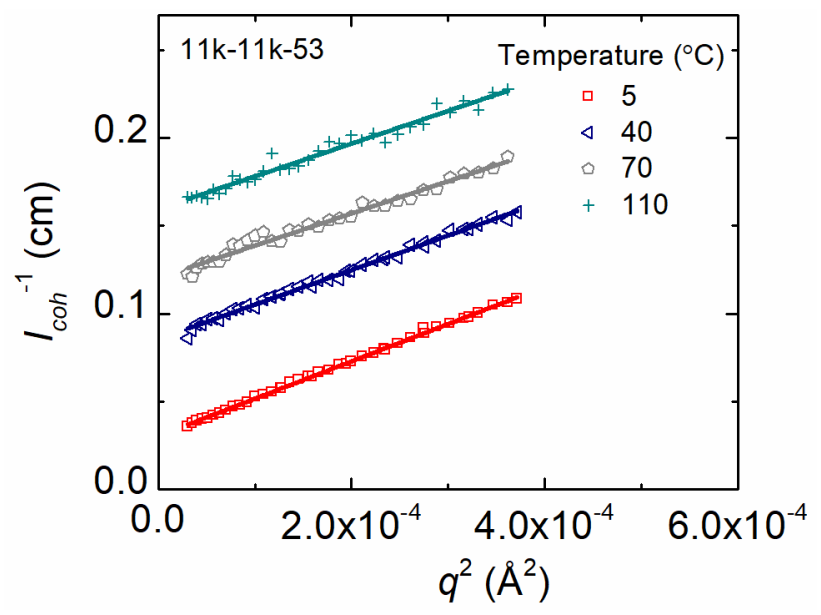

Figure S19: Zimm plot $I_{c o h}{ }^{-1}$ vs. $q$ obtained for blend 11k-11k-53 at representative temperatures. 

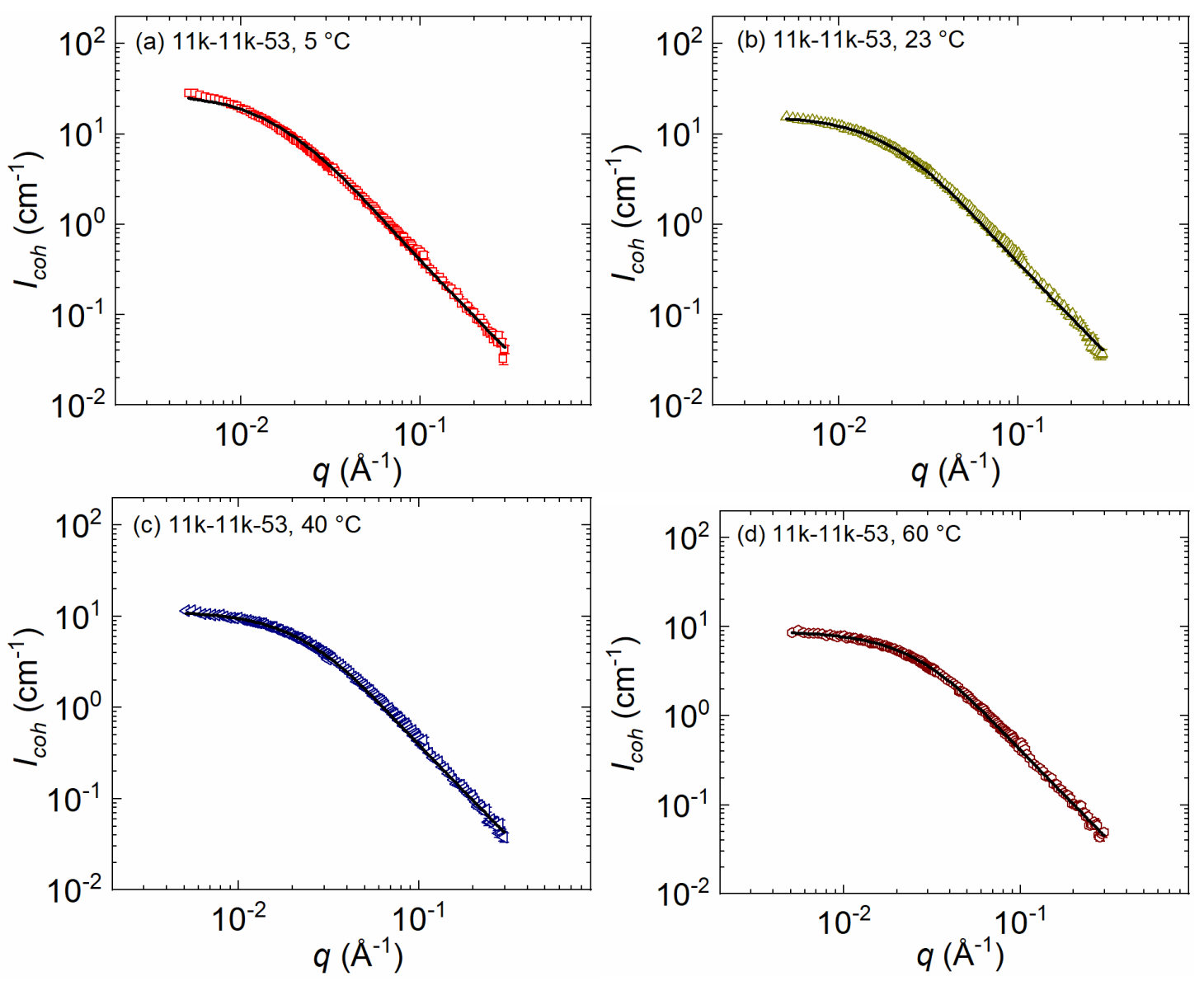

Figure S20: Representative SANS data obtained from blend 11k-11k-53. The Random Phase Approximation was fit to the data. $\chi$ and $\alpha$ were adjustable parameters in the data fitting. The error bars represent the uncertainty in $I_{c o h}$. In many cases, error bars are smaller than the data points and not shown. 


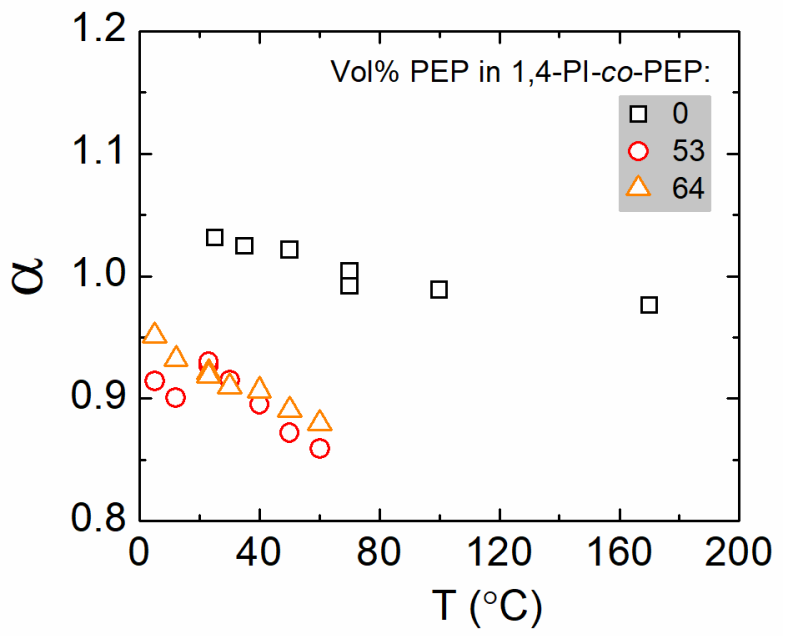

Figure S21: The chain expansion factor, $\alpha$, obtained from RPA fitting, was plotted as a function of temperature for the 1,4-PI-co-PEP / PEP blends with PEP content varying from $0-64 \mathrm{vol} \%$. $\alpha$ was calculated using literature values of $l_{i}=5.7$ and $6.9 \AA$ for 1,4-PI and PEP respectively, based on $v_{\text {ref }}=100 \AA^{3}$. 
Table S7: $\chi$ extracted through Zimm Analysis and RPA Fitting and $\alpha$ Extracted from RPA Fitting for 1,4-PI-co-PEP / PEP Blends

(a) Blend $5 \mathrm{k}-2 \mathrm{k}-0$

\begin{tabular}{|l|l|l|l|l|l|l|l|}
\hline $\mathrm{T}\left({ }^{\circ} \mathrm{C}\right)$ & 25 & 35 & 50 & 70 & 70 & 100 & 170 \\
\hline$\chi_{\mathrm{Zimm}^{\mathrm{a}}}$ & $0.0316 \pm$ & $0.0293 \pm$ & $0.0267 \pm$ & $0.0265 \pm$ & $0.0231 \pm$ & $0.0170 \pm$ & $0.0332 \pm$ \\
& 0.0016 & 0.0015 & 0.0013 & 0.0013 & 0.0012 & 0.0008 & 0.0017 \\
\hline$\chi_{\mathrm{RPA}^{\mathrm{b}}}$ & $0.0330 \pm$ & $0.0315 \pm$ & $0.0294 \pm$ & $0.0267 \pm$ & $0.0265 \pm$ & $0.0232 \pm$ & $0.0174 \pm$ \\
& 0.0026 & 0.0022 & 0.0021 & 0.0019 & 0.0019 & 0.0016 & 0.0012 \\
\hline$\alpha^{\mathrm{c}}$ & 1.09 & 1.08 & 1.08 & 1.04 & 1.06 & 1.04 & 1.03 \\
\hline
\end{tabular}

(b) Blend $11 \mathrm{k}-11 \mathrm{k}-53$

\begin{tabular}{|l|l|l|l|l|l|l|l|l|}
\hline $\mathrm{T}\left({ }^{\circ} \mathrm{C}\right)$ & 5 & 12 & 23 & 23 repeat & 30 & 40 & 50 & 60 \\
\hline$\chi_{\mathrm{Zimm}^{\mathrm{a}}}$ & $0.0087 \pm$ & $0.0083 \pm$ & $0.0079 \pm$ & $0.0079 \pm$ & $0.0076 \pm$ & $0.0073 \pm$ & $0.0070 \pm$ & $0.0066 \pm$ \\
& 0.0005 & 0.0005 & 0.0005 & 0.0005 & 0.0005 & 0.0004 & 0.0004 & 0.0004 \\
\hline$\chi_{\mathrm{RPA}^{\mathrm{b}}}$ & $0.0085 \pm$ & $0.0082 \pm$ & $0.0078 \pm$ & $0.0078 \pm 0$. & $0.0076 \pm$ & $0.0072 \pm$ & $0.0069 \pm$ & $0.0066 \pm$ \\
& 0.0006 & 0.0006 & 0.0005 & 0005 & 0.0005 & 0.0005 & 0.0005 & 0.0005 \\
\hline$\alpha^{\mathrm{c}}$ & 0.99 & 0.97 & 1.00 & 1.00 & 0.99 & 0.97 & 0.94 & 0.93 \\
\hline
\end{tabular}

\begin{tabular}{|l|l|l|l|l|}
\hline $\mathrm{T}\left({ }^{\circ} \mathrm{C}\right)$ & 60 repeat & 70 & 100 & 110 \\
\hline$\chi_{Z \mathrm{iimm}}{ }^{\mathrm{a}}$ & $0.0066 \pm 0.0004$ & $0.0064 \pm 0.0004$ & $0.0057 \pm 0.0003$ & $0.0055 \pm 0.0003$ \\
\hline$\chi_{\mathrm{RPA}^{\mathrm{b}}}{ }^{\mathrm{a}}$ & NA & NA & NA & NA \\
\hline$\alpha^{\mathrm{C}}$ & NA & NA & NA & NA \\
\hline
\end{tabular}

(c) Blend 24k-11k-64

\begin{tabular}{|c|c|c|c|c|c|c|c|c|c|c|}
\hline $\mathrm{T}\left({ }^{\circ} \mathrm{C}\right)$ & 5 & 12 & \multicolumn{2}{|c|}{23} & 23 repeat & 30 & \multicolumn{2}{|l|}{40} & 50 & 60 \\
\hline$\chi_{\text {Zimm }^{a}}$ & $\begin{array}{l}0.0048 \pm \\
0.0003\end{array}$ & $\begin{array}{l}0.0047 \pm \\
0.0003\end{array}$ & \multicolumn{2}{|c|}{$\begin{array}{l}0.0044 \pm \\
0.0003\end{array}$} & $\begin{array}{l}0.0044 \pm \\
0.0003\end{array}$ & $\begin{array}{l}0.0042 \pm \\
0.0003\end{array}$ & \multicolumn{2}{|c|}{$\begin{array}{l}0.0041 \pm \\
0.0002\end{array}$} & $\begin{array}{l}0.0039 \pm \\
0.0002\end{array}$ & $\begin{array}{l}0.0037 \pm \\
0.0002\end{array}$ \\
\hline$\chi_{\mathrm{RPA}^{\mathrm{b}}}$ & $\begin{array}{l}0.0048 \pm \\
0.0003\end{array}$ & $\begin{array}{l}0.0046 \pm \\
0.0003\end{array}$ & \multicolumn{2}{|c|}{$\begin{array}{l}0.0044 \pm \\
0.0003\end{array}$} & $\begin{array}{l}0.0044 \pm 0 . \\
0003\end{array}$ & $\begin{array}{l}0.0042 \pm \\
0.0003\end{array}$ & \multicolumn{2}{|c|}{$\begin{array}{l}0.0041 \pm \\
0.0003\end{array}$} & $\begin{array}{l}0.0039 \pm \\
0.0003\end{array}$ & $\begin{array}{l}0.0037 \pm \\
0.0003\end{array}$ \\
\hline$\alpha^{\mathrm{c}}$ & 1.03 & 1.01 & \multicolumn{2}{|c|}{1.00} & 0.99 & 0.98 & \multicolumn{2}{|c|}{0.98} & 0.96 & 0.97 \\
\hline & $\mathrm{T}\left({ }^{\circ} \mathrm{C}\right)$ & \multicolumn{2}{|l|}{60 repeat } & \multicolumn{2}{|c|}{70} & 100 & & \multicolumn{2}{|c|}{110} & \\
\hline & $\chi_{\text {Zimm }^{a}}$ & \multicolumn{2}{|c|}{$0.0037 \pm 0.0002$} & \multicolumn{2}{|c|}{$0.0036 \pm 0.0002$} & \multicolumn{2}{|c|}{$0.0032 \pm 0.0002$} & \multicolumn{2}{|c|}{$0.0031 \pm 0.0002$} & \\
\hline & $\chi_{\mathrm{RPA}^{\mathrm{b}}}$ & \multicolumn{2}{|c|}{ NA } & \multicolumn{2}{|c|}{ NA } & \multicolumn{2}{|c|}{ NA } & \multicolumn{2}{|c|}{ NA } & \\
\hline & $\alpha^{\mathrm{c}}$ & \multicolumn{2}{|l|}{ NA } & \multicolumn{2}{|c|}{ NA } & \multicolumn{2}{|l|}{ NA } & \multicolumn{2}{|c|}{ NA } & \\
\hline
\end{tabular}

${ }^{a}$ Error represents the uncertainty in $\chi$, calculated by propagating error sources together as described in main text.

${ }^{\mathrm{b}}$ For some temperatures, the SANS data were only acquired at low- $q$ configuration so that $q$ range is not sufficient to quantify the chain dimenssions through RPA fitting, and NA was labeled. Error represents the uncertainty in $\chi$, calculated by propagating error sources together as described in main text.

${ }^{\mathrm{c}}$ The chain expansion factor, $\alpha$, obtained from RPA was calculated using literature values of $l i=$ 5.7 and $6.9 \AA$ for 1,4-PI and PEP respectively, based on $v_{\text {ref }}=100 \AA^{3} .^{7}$ For some temperatures where only low- $q$ configuration data were acquired and not sufficient for RPA model fitting to extract $\alpha$, NA was labled. 


\section{Calculation of Blend Critical Composition}

The critical compositions of the blends were determined as

$$
\phi_{1, c}=\frac{1}{1+\sqrt{\frac{N_{1}}{N_{2}}}}
$$

\section{$\underline{\text { Rescaling Literature } \chi \text { Values to a Reference Volume of } 100 \AA^{3}}$}

1. Bates, F. S.; Fetters, L.; Wignall, G., Thermodynamics of isotopic polymer mixtures: poly (vinylethylene) and poly (ethylethylene). Macromolecules 1988, 21 (4), 1086-1094.

$\chi(\mathrm{T})$ for an isotopic blend containing PEE and perdeuterated PEE was reported based on a reference volume of $v_{\text {ref,lit }}=v_{H} \phi_{H}+v_{D} \phi_{D}$, where $v_{H}$ and $v_{D}$ denote the monomer volume of hydrogenous and deuterated species respectively and $\phi_{H}$ and $\phi_{D}$ represent the volume fraction of the hydrogenous and deuterated species respectively. $v_{\text {ref,lit }}$ was calculated at each measurement temperature based on the segment volume and a thermal expansion coefficient of $0.0006 / \mathrm{K}$. At $23{ }^{\circ} \mathrm{C}, v_{\text {ref,lit }}$ was calculated to be $107 \AA^{3} \cdot \chi(\mathrm{T})$ of PEE isotopic blend based on $v_{\text {ref, }, \text { it }}=v_{H} \phi_{H}+$ $v_{D} \phi_{D}$ was reported as $\chi_{\text {lit }}=\frac{0.279}{T}-0.00004 .^{4} \chi(\mathrm{T})$ was normalized to our reference volume, $v_{r e f}=100 \AA^{3}$, using $\chi=\chi_{l i t} v_{r e f, l i t} / v_{r e f}$ where $\chi_{\text {lit }}$ and $v_{r e f, l i t}$ are the literature values and $v_{r e f}$ $=100 \AA^{3}$. The result was $\chi=\frac{0.3036}{T}-0.0002$.

In our study, the appropriate isotopic blend to use for comparison purposes is a blend of PEE and partially deuterated PEE (since we did not use perdeuterated PEE in our study). Therefore, we used the $\chi$ of PEE / perdeuterated PEE discussed above to calculate the $\chi$ of PEE / partially deuterated PEE:

$\chi_{P E E / \text { partially deuterated } P E E}=\left(\frac{n_{D}}{8}\right)^{2} \chi_{P E E / \text { perdeuterated } P E E}$

where $n_{D}$ is the number of deuterium atoms per repeat unit in the partially deuterated PEE (average value of all of our samples was 2.05). The $\chi(\mathrm{T})$ of PEE / partially deuterated PEE based on $v_{r e f}=$ 
$100 \AA^{3}$ was therefore determined as $\chi=\frac{0.0190}{T}-0.00001$ and is plotted in Figure 4 of the main text.

2. Gehlsen, M. D.; Rosedale, J. H.; Bates, F. S.; Wignall, G. D.; Hansen, L.; Almdal, K., Molecular weight scaling in critical polymer mixtures. Physical Review Letters 1992, 68 (16), 2452.

$\chi(\mathrm{T})$ for an isotopic blend containing PEP and deuterated PEP with $n_{D}$ ranging from 2.86 to 9.9 was measured based on $v_{r e f, l i t}=136 \AA^{3}$ at $23{ }^{\circ} \mathrm{C}$. A thermal expansion coefficient of 0.00075/K was used to account for the temperature dependence of the reference volume. Based on $v_{r e f, l i t}, \chi(\mathrm{T})$ of PEP isotopic blend was reported as $\chi_{\text {lit }}=\left(\frac{n_{D}}{10}\right)^{2}\left(\frac{0.571}{T}-0.000656\right) .{ }^{8} \mathrm{We}$ rescaled $\chi(\mathrm{T})$ of PEP isotopic blend to $v_{\text {ref }}=100 \AA^{3}$, using $\chi=\chi_{\text {lit }} v_{\text {ref,lit }} / v_{\text {ref }}$, and the result was $\chi=\left(\frac{n_{D}}{10}\right)^{2}\left(\frac{0.4426}{T}-0.0006\right)$. We applied an average $n_{D}$ of 3.16 in our system and plotted the PEP isotopic $\chi(T)$ as the dotted line $\left(\chi=\frac{0.0442}{T}-0.00006\right)$ in Figure 7 of the main text.

3. Bates, F. S.; Rosedale, J. H.; Stepanek, P.; Lodge, T. P.; Wiltzius, P.; Fredrickson, G. H.; Hjelm Jr, R. P., Static and dynamic crossover in a critical polymer mixture. Physical Review Letters 1990, 65 (15), 1893.

The $\chi(\mathrm{T})$ of a blend containing 1,4-PI / partially deuterated PEP was measured based on a temperature-independent $v_{r e f, l i t}=130 \AA^{3}$, resulting in $\chi_{\text {lit }}=\frac{26.5}{T}-0.0367 .{ }^{9}$ We rescaled $\chi(\mathrm{T})$ of 1,4-PI / partially deuterated PEP to $v_{\text {ref }}=100 \AA^{3}$, using $\chi=\chi_{\text {lit }} v_{\text {ref }, \text { lit }} / v_{\text {ref }}$, resulting in $\chi=$ $\left(\frac{20.38}{T}-0.0282\right)$, which is plotted as the dashed line in Figure 7 of the main text. 


\section{References}

1. $\quad$ Qiu, J.; Mongcopa, K. I.; Han, R.; López-Barrón, C. R.; Robertson, M. L.;

Krishnamoorti, R., Thermodynamic interactions in a model polydiene/polyolefin blend based on 1,2-polybutadiene. Macromolecules 2018, 51 (8), 3107-3115.

2. Grant, D. M.; Paul, E. G., Carbon-13 magnetic resonance. II. Chemical shift data for the alkanes. Journal of the American Chemical Society 1964, 86 (15), 2984-2990.

3. Pretsch, E.; Clerc, T.; Seibl, J.; Simon, W., Tabellen zur Strukturaufklärung organischer Verbindungen: mit spektroskopischen Methoden. Springer-Verlag: 2013; Vol. 15.

4. $\quad$ Bates, F. S.; Fetters, L.; Wignall, G., Thermodynamics of isotopic polymer mixtures: poly (vinylethylene) and poly (ethylethylene). Macromolecules 1988, 21 (4), 1086-1094.

5. Roovers, J.; Toporowski, P., Microheterogeneity in miscible blends of 1, 2-polybutadiene and 1, 4-polyisoprene. Macromolecules 1992, 25 (13), 3454-3461.

6. $\quad$ Krishnamoorti, R., PhD Thesis. Princeton University 1994.

7. Mark, J. E., Physical properties of polymers handbook. Springer: 2007; Vol. 1076.

8. Gehlsen, M. D.; Rosedale, J. H.; Bates, F. S.; Wignall, G. D.; Hansen, L.; Almdal, K., Molecular weight scaling in critical polymer mixtures. Physical Review Letters 1992, 68 (16), 2452.

9. $\quad$ Bates, F. S.; Rosedale, J. H.; Stepanek, P.; Lodge, T. P.; Wiltzius, P.; Fredrickson, G. H.; Hjelm Jr, R. P., Static and dynamic crossover in a critical polymer mixture. Physical Review Letters 1990, 65 (15), 1893. 